\title{
Can Intonational Phrase Structure Be Primed (Like Syntactic Structure)?
}

\author{
Kristen M. Tooley \\ University of Illinois at Urbana-Champaign and Beckman \\ Institute, Urbana, Illinois
}

\author{
Agnieszka E. Konopka \\ Max Planck Institute for Psycholinguistics, Nijmegen, the \\ Netherlands, and Donders Center for Brain, Cognition, and \\ Behavior, Nijmegen, the Netherlands
}

\author{
Duane G. Watson \\ University of Illinois at Urbana-Champaign and Beckman Institute, Urbana, Illinois
}

\begin{abstract}
In 3 experiments, we investigated whether intonational phrase structure can be primed. In all experiments, participants listened to sentences in which the presence and location of intonational phrase boundaries were manipulated such that the recording included either no intonational phrase boundaries, a boundary in a structurally dispreferred location, a boundary in a preferred location, or boundaries in both locations. In Experiment 1, participants repeated the sentences to test whether they would reproduce the prosodic structure they had just heard. Experiments 2 and 3 used a prime-target paradigm to evaluate whether the intonational phrase structure heard in the prime sentence might influence that of a novel target sentence. Experiment 1 showed that participants did repeat back sentences that they had just heard with the original intonational phrase structure, yet Experiments 2 and 3 found that exposure to intonational phrase boundaries on prime trials did not influence how a novel target sentence was prosodically phrased. These results suggest that speakers may retain the intonational phrasing of a sentence, but this effect is not long-lived and does not generalize across unrelated sentences. Furthermore, these findings provide no evidence that intonational phrase structure is formulated during a planning stage that is separate from other sources of linguistic information.
\end{abstract}

Keywords: prosody, syntactic priming, intonational phrase boundary

When a person decides to speak, a number of processes must unfold to produce an utterance that is well formed and comprehensible by the listener. Evident to most is the necessity of deciding on a message and then choosing the words that adequately convey that message. What is less obvious but just as critical to successful communication is deciding how those words should be combined together given the rules of the language and how those words are grouped together in time during overt production. The former falls under the domain of syntax, while the latter falls under the domain of prosodic phrasing.

The representation of these two aspects of spoken language has been an area of considerable interest in the language-processing

This article was published Online First November 4, 2013.

Kristen M. Tooley, Department of Psychology, University of Illinois at Urbana-Champaign, and Beckman Institute, Urbana, Illinois; Agnieszka E. Konopka, Max Planck Institute for Psycholinguistics, Nijmegen, the Netherlands, and Donders Center for Brain, Cognition, and Behavior, Nijmegen, the Netherlands; Duane G. Watson, Department of Psychology, University of Illinois at Urbana-Champaign, and Beckman Institute, Urbana, Illinois.

This work was supported by National Institutes of Health Grants R01 DC008774 and T32 HD055272 and by the James S. McDonnell Foundation.

Correspondence concerning this article should be addressed to Kristen M. Tooley, who is now at the Department of Psychology, Texas State University, 601 University Drive, San Marcos, TX 78666. E-mail: ktooley@txstate.edu literature. In recent decades, numerous studies investigating syntactic repetition have helped to illuminate the nature of the processes responsible for syntactic assembly and have offered explanations as to how these processes might interact with other types of linguistic information (Bock, 1986; Chang, Dell, \& Bock, 2006; Pickering \& Branigan, 1998; and others; see Pickering \& Ferreira, 2008 , for a review of syntactic priming effects). The current study seeks to investigate the formulation of prosodic phrase structure using a methodology analogous to that used to investigate formulation of syntactic structure. Specifically, can prosodic phrase structure be primed in a manner similar to syntactic structure? Evaluating how primeable prosodic phrase structure might be can inform theories of prosodic representation in much the same way that priming studies on syntactic structure have informed theories of syntactic representation, that is, by revealing whether or not prosodic phrasing is generated by means of a mechanism that is independent of syntactic and lexical processing.

\section{The Nature of Prosodic Structure}

Prosody is typically used to refer to the aspects of the acoustic signal that are independent of the lexical items in the sentence, such as rhythm, stress, pitch, intonation, and speaking rate. In this article, we investigate only one aspect of prosody, intonational phrasing, which refers to how speakers group words together in time. Intonational phrases tend to be delimited from other phrases by intonational boundaries: These boundaries are correlated with the percept of a pause and sometimes coincide with one, but they 
can also be signaled by a lengthening of the preboundary word and tonal movement at the end of the phrase.

Although intonational phrasing is influenced by a number of different factors, syntax and semantics are both known to have clear effects on how words are grouped together (e.g., Gee \& Grosjean, 1983; Schafer, 1997; Selkirk, 1984; Truckenbrodt, 1999; Watson \& Gibson, 2004). For example, speakers tend to produce intonational phrase boundaries, usually marked by a discernible pause, at the beginnings and ends of syntactic phrases (Breen, Watson, \& Gibson, 2011; Watson \& Gibson, 2005), which suggests that intonational phrase boundaries are closely linked to syntactic and semantic planning (see Ferreira, 1991; Schafer, 1997; Watson \& Gibson, 2004; Wheeldon \& Lahiri, 1997). A systematic link between intonational phrases and syntax is also evident during comprehension, where intonational phrasing can facilitate selection of a syntactic interpretation in syntactically ambiguous sentences (see Wagner \& Watson, 2010, for a review). The extent to which a speaker's choice of intonational phrasing is determined by syntax is an open debate, however. Theories span the spectrum from the extreme view that intonational phrase structure and syntactic structure are one and the same (Steedman, 1991) to the view that syntax is one of many constraints that influence how a sentence is intonationally phrased (see Shattuck-Hufnagel, \& Turk, 1996).

One problem for a strict one-to-one view of the relationship between syntactic and intonational phrase structure is the finding that speakers can (and do) produce more than one acceptable intonational phrasing for a given syntactic structure. For example (from Shattuck-Hufnagel \& Turk, 1996), the sentence "Rebecca won their support" can be acceptably produced with one of two intonational phrasings (either "Rebecca // won their support" or "Rebecca won // their support," where // signifies an intonational boundary). Furthermore, not all ambiguities can be disambiguated by means of intonational phrasing. Intonational phrasing is most helpful in distinguishing between syntactic alternatives when those alternatives contain syntactic constituents that differ in their surface-level relations. For example, in an attachment ambiguity like "Kristen saw the student with the monocle," either Kristen has the monocle or the student does. A boundary after "student" encourages the former interpretation, while a boundary after "saw" encourages the latter. In contrast, ambiguities that do not depend on surface-level attachment differences, such as the classic gardenpath sentence "The horse raced past the barn fell," are typically not disambiguated prosodically (see Wagner \& Watson, 2010, for a review of which structures are affected by intonational phrasing). These types of data suggest that intonational phrasing and syntactic structure may be generated at distinct levels of representation. Further evidence for the abstractness of intonational phrase structures comes from work showing that intonational phrasing can constrain the application of phonological sandhi rules, like stress shift in English (Nespor \& Vogel, 1986), and that intonational phrase boundaries can serve as abstract slots into which pauses and lexical items are inserted (Ferreira, 1993).

Yet, despite the evidence that intonational phrasing is itself a distinct level of representation, relatively little is known about the processes that are involved in constructing intonational phrase structures in language production. One open question is whether there are procedures or heuristics that construct prosodic represen- tations independent of the syntactic, semantic, and lexical aspects of the sentence.

A similar debate took place in psycholinguistics with respect to the nature of syntactic processing (see Bock, 1982, for a review), where functional approaches to syntax questioned whether language production involved a processing stage in which syntactic information was represented separately (or independently) from other types of linguistic information. Research on syntactic priming in production (and more recently in comprehension) has since provided strong support for the notion that speakers indeed construct purely syntactic representations when planning and processing full sentences. We turn now to a review of these findings and highlight their applicability to the question of isolable intonational phrase structure formulation in production.

\section{Findings From Syntactic Priming Studies}

Prior to Bock's (1986) original demonstration of syntactic priming across unrelated sentences, some theorists questioned the existence of a separate stage of syntactic processing during language production (Bates \& MacWhinney, 1982). These accounts suggested that structural information and semantic and/or conceptual information continuously interacted during grammatical encoding, obviating the necessity for a purely syntactic stage of processing. A contrasting view posited a separate processing stage for syntax during which sentence structure is assembled with abstract structural mechanisms that do not immediately rely on, or interact with, conceptual or semantic sources of information (Garrett, 1980).

Bock's (1986) study provided support for the latter view by showing that it was possible to prime syntactic constructions while holding other linguistic factors (e.g., words, sentence meaning, and noun animacy) constant. Participants in this study were asked to repeat sentences and describe pictures under the guise of a memory test. On prime trials, they heard the experimenter produce an active or passive description of a pictured event and repeated this sentence out loud; on target trials, they generated their own descriptions for new pictures. Participants reliably repeated the syntax of sentences they had recently heard when describing the target pictures. For example, following an active-voice prime sentence like "One of the fans punched the referee," they were more likely to then describe a target picture of lightning striking a church with an active-voice sentence (like "Lightning struck the church") than when they had first heard a passive prime. The finding that exposure to a particular structure can influence future choice of syntactic structure in an unrelated utterance strongly supports the notion that syntactic processing does unfold, at least initially, independently from processes unfolding at other levels of representation.

Numerous replications and extensions of this study over the past 3 decades have contributed to theories of both production and comprehension. They have generated debates, for example, as to whether such repetition effects reflect adaptation in the language system in the form of implicit learning (e.g., Chang et al., 2006, and others) or whether they are the product of system-wide coordination between speakers (Garrod \& Pickering, 2004). Across theories, the fundamental claims about the nature of structural assembly remain the same: Sentence structure can be generated by abstract processing mechanisms that set the stage for subsequent 
processes (such as lexical retrieval and insertion processes) when planning an utterance.

In this study, we apply a priming paradigm to investigate whether a similar processing mechanism exists for planning of intonational phrase structure. Our logic here is the same as the logic that underlay Bock's (1986) experiment: If an abstract, independent prosodic representation is engaged in language production, than it should be amenable to priming. Recent theories of communication and priming, specifically alignment theory (Garrod \& Pickering, 2004), argue that priming occurs at every level of linguistic representation and that it does so to facilitate communication between interlocutors. If priming of intonational phrase boundaries does not occur, either there is no abstract, independent level of representation for intonational phrase planning in production or priming is not as ubiquitous as previously thought and proposals like the alignment theory must be reevaluated. We explore these questions below.

\section{Can Intonational Phrase Structure Be Primed?}

Taking an approach analogous to that of syntactic priming studies, we tested whether it is possible to prime an intonational phrase structure (the placement of intonational phrase boundaries) across unrelated sentences. We used a task adapted from syntactic priming paradigms where speakers are exposed to a particular syntactic structure on prime trials and may reuse the same structure on the following target trial. On the assumption that generalization of structure from one sentence to another unrelated sentence provides evidence for priming of an abstract structure, we tested whether exposure to sentences with different intonational phrase boundaries influenced the intonational phrasing of participants' own speech.

To our knowledge, there are no process models of how intonational phrase boundaries are formulated in language production. Part of the challenge lies in the fact that the distribution of intonational boundaries is determined by multiple levels of linguistic structure, including pragmatics, discourse, syntax, semantics, and phonology (see Shattuck-Hufnagel \& Turk, 1996, for a review). Standard models of production posit that production occurs in several stages that run in parallel (e.g., Bock \& Levelt, 1994). At the highest level, the message to be produced is formulated. At the next stage, grammatical structure is formulated, and lexical items are selected. In the final stage of production, an articulatory motor plan is formulated and executed. Given the fact that intonational boundary placement can be influenced by processing at multiple levels of representation and impacts how segments are acoustically realized, finding a place for the generation of prosodic boundaries in the system remains a puzzle.

However, given these links between prosody and other aspects of linguistic representation, we can ask a very basic question about how prosodic boundaries are formulated in production by using a priming paradigm. There are at least two ways in which the production system might formulate boundaries. In the first, processing at the message and formulation levels determines boundary presence through an intermediate prosodic boundary formulation stage that is independent of other levels of representation. This is outlined in Figure 1. For example, if the semantics of a sentence require a boundary as in the case of an aside or vocative or if processing at the formulation stage requires a boundary to buy

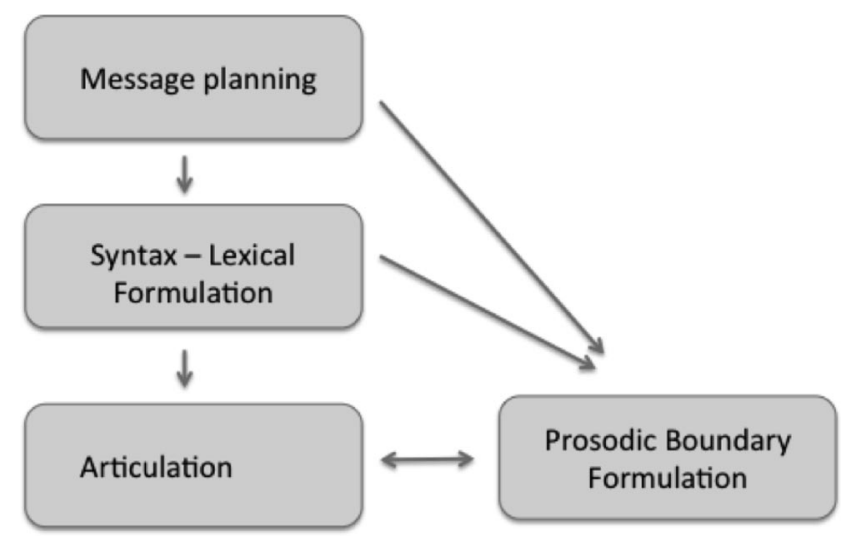

Figure 1. Model of prosody production in which there is an abstract prosodic boundary formulator.

more time for processing (e.g., Breen et al., 2011; Watson \& Gibson, 2004), a signal is sent to the prosodic planning system, which, in coordination with the articulation system, formulates and executes a prosodic boundary. This abstract prosodic formulator might possibly contain representations of syntactic or semantic constraints on boundary placement as well as mechanisms for determining ideal locations in an utterance to initiate a boundary. If this abstract prosodic boundary formulation stage exists, we would expect its representations to be amenable to priming.

A second possibility is that there is no abstract prosodic boundary formulator. Production processes at the message and formulation levels directly interface with articulation, which contains phonological and phonetic plans for implementing boundaries. This is illustrated in Figure 2. When the need for a boundary arises for either semantic or syntactic reasons, a signal is sent directly to the articulation system, which initiates the production of a boundary. This signal carries no information about the syntactic structure itself and includes only a "go" signal for a boundary to be initiated. Critically, such an architecture predicts that prosodic boundaries at certain syntactic and semantic locations should not prime because there is no representation tracking the relationship between syntactic structure and prosody to be primed.

We tested the question of abstractness of the prosodic boundary formulator in three experiments. In all experiments, participants listened to recorded sentences that contained no intonational boundaries and sentences that contained intonational phrase boundaries spliced into two different syntactic locations (a preferred syntactic location, a dispreferred syntactic location, or both locations). In Experiment 1, participants were asked only to repeat back the sentences they had just heard. Experiments 2 and 3 used a prime-target manipulation. Participants listened to recorded sentences (primes) and read sentences printed on the screen (targets) on alternating trials. In Experiment 2, they repeated each recorded prime sentence before moving on to the target trial; they then silently read the target sentence and were prompted to repeat it out loud from memory. In Experiment 3, participants were not asked to repeat the prime sentence but proceeded immediately to the target trial.

Our paradigm is adapted from the rapid serial visual presentation (RSVP) paradigm used successfully to detect syntactic prim- 


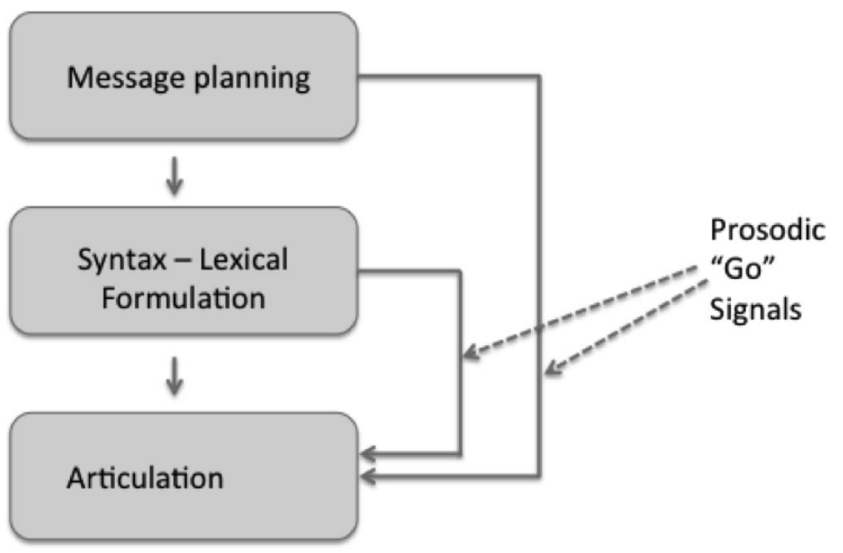

Figure 2. Model of prosody production in which the message and structural formulation systems send signals directly to the articulator to trigger the production of an intonational boundary.

ing effects in production. This paradigm involves showing participants an RSVP of a prime sentence that the participants must (after a brief distractor task) repeat back from memory (Potter \& Lombardi, 1998). The participants' repetition of this prime sentence becomes the priming production episode. A subsequent RSVP sentence is the target sentence. Rapid presentation of the sentences reduces the likelihood of participants forming a verbatim trace of the original sentence and thus requires that they reconstruct, rather than repeat, this sentence from memory when prompted to do so after the distractor task. Participants' reconstructions of the target sentences are examined for persistence of the syntactic structure from the priming episode. What is manipulated is whether or not the syntactic structure of the RSVP prime sentence matches that of the RSVP target sentence. RSVP studies have shown that participants are more likely to repeat the target sentence with the original structure when this structure matches the syntax of the prime sentence (e.g., repeating a prepositional object target like She gave a flower to the boy with the prepositional dative structure if the prime sentence also used this structure) and, conversely, are more likely to produce a target sentence with the structure of the prime sentence when the syntax of the primes and targets does not match (e.g., repeating a prepositional object target like She gave a flower to the boy with double-object dative syntax if the prime sentence also used the double-object structure). These findings indicate that the syntactic processing related to producing/ reconstructing the prime sentence primes the processing related to producing/reconstructing the target sentence, making it more likely that the primed syntactic form would be used to convey the message presented in the target sentence. This paradigm has successfully replicated syntactic priming effects that were originally observed using Bock's (1986) sentence repetition and picture description paradigm and has been used to extend syntactic priming findings to novel contexts, such as priming idioms (Konopka \& Bock, 2009) and datives (Tooley \& Bock, 2013).

For present purposes, one potential concern may be that message and production planning systems might be engaged in repetition or reading to a lesser extent than they are in spontaneous speech, complicating an investigation of the abstractness of prosodic representations. Admittedly, our paradigm relies on compre- hension of the intonational phrase information as the priming episode, rather than on production, which differs somewhat from Potter and Lombardi's (1998) original paradigm. However, Potter and Lombardi observed significant priming whether the prime sentence was read and repeated or just read (i.e., not produced). In a different task, Bock, Dell, Chang, and Onishi (2007) explicitly investigated this question by comparing priming effects from an experiment where speakers comprehended and repeated a recorded prime sentence (Griffin \& Bock, 2000) and an experiment where they only comprehended the prime sentence. The magnitude of the priming effects in these two conditions was not reliably different, indicating that priming episodes that rely on comprehension processes are just as successful at influencing subsequent production processes as production priming episodes. Other syntactic priming studies have also found reliable effects when the priming information was comprehended, such as when conversation partners prime each other's produced syntactic structures based on the structure they have heard the other say (e.g., Branigan, Pickering, \& Cleland, 2000; Cleland \& Pickering, 2003). Relevant to the current experiment, these findings reveal that quickly reading/ comprehending sentences engages language production processes similar to those implicated in sentence formulation in spontaneous speech, such as generating a message-level representation and conveying that message through a choice of syntactic structure. Thus, using comprehended intonational phrase boundaries as the source of boundary priming is a viable approach to investigating priming for this type of representation.

In addition, previous studies of boundary placement that used similar paradigms (Breen et al., 2011; Ferreira, 1991, 1993; Gee \& Grosjean, 1983; Watson \& Gibson, 2004) have found that syntactic and semantic constraints determine boundary placement even when sentences are read out loud or recalled, rather than generated from beginning to end by participants themselves. Participants place boundaries at major syntactic constituents and disprefer placing boundaries between semantically linked words in these tasks. Thus, the literature suggests that these types of paradigms, though they admittedly differ from spontaneous speech on a number of dimensions, must engage some level of syntactic and semantic formulation for these factors to influence boundary placement.

In the experiments we report below, the repeated sentences were perceptually coded for the presence of an intonational phrase boundary at the manipulated syntactic locations. Because intonational boundaries are highly correlated with increased lengthening of the preboundary word as well as a perceived pause at the boundary, we also measured the word-and-pause durations from the onset of the word prior to the manipulated boundary location through the onset of the word following that location.

We compared production of intonational phrase boundaries in the preferred and dispreferred syntactic locations of each repeated sentence when participants had just heard a sentence with a boundary in that location to when they had not. Increased production of boundaries at the locations that coincide with prime sentence boundary locations could suggest abstract priming of intonational phrase structure. In turn, this would support an account of prosodic representation where intonational phrasing processes operate independently of other (nonprosodic) sources of information during sentence formulation. Finding little difference in speakers' rates of boundary production at the primed locations would be consistent 
with a lack of priming for intonational phrase structure, and this would be inconsistent with an abstract account of prosodic formulation.

\section{Experiment 1}

Experiment 1 investigated whether a priming paradigm similar to that used in syntactic priming studies would be sufficiently sensitive to detect retention of intonational phrase structure information. Speakers in this experiment listened to and immediately repeated back the sentences they heard. The recorded sentences either had no intonational phrase boundaries or had boundaries spliced in at either a syntactically preferred location, at a syntactically dispreferred location, or at both locations, for a total of four conditions. We tested whether participants would repeat the same intonational phrasing (placement of intonational phrase boundaries) when producing these sentences from memory, even if this resulted in the production of a boundary in a dispreferred syntactic location.

\section{Method}

Participants. Forty-four students from the University of Illinois, all native speakers of English, participated in the experiment for course credit.

Materials. The sentences were obtained from filler sentences used by Tooley, Traxler, and Swaab (2009) and consisted of 20 sentences with relative clauses (e.g., The dog that pawed the door needed to be let out) and 20 sentences with main clauses (e.g., The mobster shot the men for their disloyalty; see the Appendix).

All sentences were initially recorded by a native speaker of English with and without intonational phrase boundaries in the critical regions. The boundary locations followed the first verb and the second noun of the sentence (e.g., The dog that pawed // the door // needed to be let out). Conditions with boundaries following the first verb (pawed) were considered dispreferred. Most models of boundary placement predict that intonational boundaries are less likely to occur between verbs and direct objects than in other locations (e.g., Cooper \& Paccia-Cooper, 1980; Nespor \& Vogel, 1986; Watson, Breen, \& Gibson, 2006; Watson \& Gibson, 2004), and this observation has been verified empirically (Watson et al., 2006; Watson \& Gibson, 2004). Though the dispreferred boundaries are likely to be perceived to listeners as odd, they were not so infelicitous as to cause processing difficulty or to compromise listeners' ability to comprehend or remember sentence gist. Conditions with boundaries following the second noun (door) were preferred, as the presence of a clause boundary as a well as the boundary between the noun phrase and verb phrase make this location a relatively likely one for boundary placement (e.g., Truckenbrodt, 1999; Watson \& Gibson, 2004).

From these original sentences, the experimental stimuli were created via splicing of the critical regions of each sentence, using a splicing procedure outlined below. The critical regions of each sentence comprised the word before the boundary, the boundary itself, and the word immediately following the boundary. In order to control for the prosody of regions of the sentence outside the area of interest, critical regions were spliced from recordings of each condition into a neutral carrier sentence with no intonational boundaries to create four versions of the same item: a sentence with no boundaries (the control condition), a sentence with a boundary in the syntactically dispreferred location after the first verb (The dog that pawed // the door needed to be let out), a sentence with a boundary in the syntactically preferred location after the second noun (The dog that pawed the door // needed to be let out), and a sentence with both boundaries (The dog that pawed // the door // needed to be let out). In order to control for any disruption in the acoustic contours created by the splicing procedure, each condition of the sentence contained two splices, one at each of the boundary locations outlined above. If the condition dictated that there should not be a boundary at that location, there was still a splice, but that splice was taken from the version of the sentence where the speaker did not produce a boundary at that location.

Table 1 lists the mean word-and-pause durations at the two boundary locations in sentences that included no boundaries in these locations and sentences that included boundaries at the two locations: Word-and-pause durations were longer by $450 \mathrm{~ms}$ when a boundary was present in the dispreferred location than when there was no boundary, and longer by $401 \mathrm{~ms}$ when a boundary was present in the preferred location than when there was no boundary. Word-and-pause durations in the preferred and dispreferred locations did not differ, $t(39)=.23, n s$, for sentences that did not include pauses at the two boundary locations, and $t(39)=$ $1.68, n s$, for sentences with pauses.

Four lists of stimuli were created such that each target sentence appeared in a different condition across lists. Within lists, each participant received 10 items in each condition. The target sentences were arranged such that no more than two items from the same condition followed one another, with two filler sentences occurring between any two experimental sentences. The filler sentences had various syntactic structures (e.g., cleft constructions, sentences with that-complements, sentences with fronted prepositional and temporal phrases) and were recorded by the same

Table 1

Average Word-and-Pause Durations (With Standard Deviations) in Sentences With Relative Clauses and Main Clauses When a Boundary Was and Was Not Present at the Dispreferred and Preferred Locations

\begin{tabular}{lccccc}
\hline & \multicolumn{2}{c}{ Dispreferred location } & & \multicolumn{2}{c}{ Preferred location } \\
\cline { 2 - 3 } \cline { 5 - 6 } \multicolumn{1}{c}{ Sentence type } & No boundary & Inserted boundary & & No boundary & Inserted boundary \\
\hline Sentences with relative clauses & $274(85)$ & $717(105)$ & & $278(83)$ & $662(115)$ \\
Sentences with main clauses & $296(83)$ & $752(128)$ & & $300(94)$ & $717(145)$ \\
Average of all sentences & $285(84)$ & $735(116)$ & & $289(88)$ & $690(132)$ \\
\hline
\end{tabular}


speaker. The placement of intonational phrase boundaries in the fillers was varied to ensure that the experimental stimuli and manipulation were not overly obvious to participants. One quarter of the fillers had a boundary in a syntactically dispreferred location, one quarter had a boundary in a syntactically preferred location, one quarter had boundaries in both syntactically dispreferred and preferred locations, and the final quarter had no prosodic phrase boundaries. These sentences (and boundaries) were all produced naturally by the speaker, rather than being spliced and inserted, and all participants heard the same filler sentences.

Procedure. Participants were tested individually, seated in front of a computer screen. They were told that on each trial of the experiment, they would first see the word LISTEN, signaling that they would hear a recorded sentence, followed by the word REPEAT to indicate that they should repeat this sentence out loud from memory as best they could into a microphone. The experimenter provided no explicit instructions about prosody or intonation. Participants were instructed to press the spacebar on the keyboard when they had finished repeating each sentence in order to advance the experiment to the next sentence. The first four sentences of the experiment served as practice trials.

Scoring and analyses. Participants' responses were excluded if they reconstructed the sentences with different syntax, paused for extended periods of time during production of a sentence, produced dysfluencies in the critical regions, or did not produce full sentences. Using a strict scoring criterion, sentences were only accepted if they were reproduced verbatim. Using a more lax scoring criterion, sentences with minor word changes (but no syntactic changes) were also accepted (e.g., performed the solo reconstructed as played the solo, tossed the ball reconstructed as returned the ball, summoned the painter reconstructed as saw the painter).

Two different coders scored each sentence. There is a great deal of controversy in the literature as to whether intonational boundaries are abstract linguistic categories or whether they are best thought of as a continuous, noncategorical representations (see Wagner \& Watson, 2010, for a review); thus, we used two different measures of intonational boundaries. One coder (the first author) subjectively coded for the presence of intonational boundaries, while the other (the second author) measured preboundary word length and pause length. The coders were blind to condition while coding.

The first coder scored sentences that were repeated exactly as presented and sentences that were repeated with minor wording changes in the critical regions (i.e., after applying the lax scoring criterion). This left 1,519 trials for analysis (86\% of all possible trials). The coder marked whether or not she heard an intonational boundary in each sentence in the dispreferred and preferred boundary locations (i.e., a binary dependent variable). Intonational boundaries corresponded to juncture break indices of 3 or 4 in the ToBI prosodic annotation system (Silverman et al., 1992).

The second coder measured the duration from the onset of the preboundary word up to the onset of the postboundary word in each sentence in the dispreferred and preferred boundary locations (all measurements were carried out using Praat). Since minor wording changes could affect the word-and-pause duration measure, only results obtained by applying the strict coding criterion are reported (although both scoring criteria produced similar results for this measure). Trials with word-and-pause durations lon- ger than three standard deviations away from the grand mean were excluded, leaving 1,210 trials (69\% of all trials) for the analysis of sentences repeated verbatim.

Analyses in all experiments were carried out in $\mathrm{R}$ ( $\mathrm{R}$ Development Core Team, 2012), with logit mixed models for the binary ratings of perceived intonational boundaries and linear mixedeffects models for the word-and-pause durations (Baayen, Davidson, \& Bates, 2008; Jaeger, 2008). All models treated participants and items as random effects, and random slopes were included when they improved model fit. ${ }^{1}$ Separate analyses were carried out for intonational boundaries in the dispreferred and preferred boundary locations. Prime condition was included as a fixed effect, and all tables list the contrasts between the control condition and each of the three conditions with boundaries. Effects were considered significant at $\alpha<0.05$.

\section{Results}

Production of intonational boundaries in the dispreferred location. The analysis of perceived boundaries revealed a significant effect of prime condition (see Table 2, Model a). When participants heard a sentence with a boundary after the first verb in the sentence, they tended to repeat the sentence with a boundary at that dispreferred syntactic location. This was true when they were primed with a sentence containing only one boundary at the dispreferred location and when they were primed with a sentence containing boundaries at both the dispreferred and preferred syntactic locations (see Figure 3a). These results suggest that participants did retain intonational phrase structuring information in memory and that this information influenced boundary placement in their repetitions of the recorded sentences even when those boundaries occurred at syntactically dispreferred locations.

A similar pattern was obtained with comparisons of word-andpause durations in the three conditions with intonational boundaries compared to the control condition with no boundaries (see Table 2, Model b; Figure 3b). Word-and-pause durations were longer in the dispreferred location when the recorded sentence contained a boundary in the dispreferred location, both when this was the only boundary in the sentence and when the sentence was presented with two boundaries. Word-and-pause durations in the dispreferred location did not differ from the control condition when the recorded sentence had a boundary in the preferred location.

Production of boundaries in the preferred location. The analysis of perceived boundaries in the preferred location also revealed a significant effect of prime condition (see Table 2, Model c). When participants heard a sentence with a boundary after the second noun phrase in the sentence, they tended to repeat the sentence with a boundary at that preferred syntactic location. This was true whether they were primed with a sentence contain-

\footnotetext{
${ }^{1}$ To determine whether to include random slopes in each model, random by-participant and by-item slopes for each factor were added one at a time to the simpler models run without random slopes. If inclusion of these slopes decreased the model's Akaike information criterion value relative to that of models without slopes, an analysis of variance comparison was run to verify whether the random slope reliably improved model fit. Only random slopes that at least marginally improved model fit were included in the final models.
} 
Table 2

Results of Analyses of Perceived Boundary Production and Word-and-Pause Durations in the Dispreferred and Preferred Locations of Sentences Repeated in Experiment 1

\begin{tabular}{|c|c|c|c|c|c|c|}
\hline \multirow[b]{2}{*}{ Predictor } & \multicolumn{3}{|c|}{ Boundary production } & \multicolumn{3}{|c|}{ Word-and-pause durations } \\
\hline & Estimate & $S E$ & $\overline{z \text { value }}$ & Estimate & $S E$ & $t$ value \\
\hline & \multicolumn{3}{|c|}{ Model a } & \multicolumn{3}{|c|}{ Model b } \\
\hline \multicolumn{7}{|l|}{ Dispreferred boundary location } \\
\hline Intercept (no-pause condition) & -3.13 & 0.29 & $-10.95^{*}$ & 344 & 15 & $23.33^{*}$ \\
\hline \multicolumn{7}{|l|}{ Condition contrasts } \\
\hline No pauses vs. dispreferred pause & 2.57 & 0.26 & $9.82^{*}$ & 86 & 11 & $8.02^{*}$ \\
\hline No pauses vs. preferred pause & -0.28 & 0.34 & -0.83 & 7 & 8 & 0.99 \\
\hline \multirow[t]{2}{*}{ No pauses vs. both pauses } & 1.49 & 0.27 & $5.54^{*}$ & 57 & 10 & $5.50^{*}$ \\
\hline & \multicolumn{3}{|c|}{ Model c } & \multicolumn{3}{|c|}{ Model d } \\
\hline \multicolumn{7}{|l|}{ Preferred boundary location } \\
\hline Intercept (no-pause condition) & -0.58 & 0.24 & $-2.39^{*}$ & 394 & 19 & $20.56^{*}$ \\
\hline \multicolumn{7}{|l|}{ Condition contrasts } \\
\hline No pauses vs. dispreferred pause & 0.13 & 0.17 & 0.78 & 25 & 10 & $2.60^{*}$ \\
\hline No pauses vs. preferred pause & 1.25 & 0.17 & $7.24^{*}$ & 75 & 9 & $7.99^{*}$ \\
\hline No pauses vs. both pauses & 1.00 & 0.17 & $5.90^{*}$ & 79 & 9 & $8.37^{*}$ \\
\hline
\end{tabular}

Note. In all analyses, the effect of prime condition was assessed with three contrasts that compared results from sentences presented with either pause or two pauses to the no-pause condition (intercept). Model $\mathrm{b}$ includes random by-participant slopes for the condition factor.

$* p<.05$

ing only one boundary or a sentence with boundaries at both the preferred and dispreferred syntactic locations (see Figure 3a).

Similarly, comparisons of word-and-pause durations in the three conditions with boundaries against the control condition showed that speakers produced longer durations when the recorded sentences contained a boundary in either or both locations (see Table 2, Model d; Figure 3b). Durations were found to be longer in all conditions where the recorded sentence contained a boundary, but as expected, this effect was strongest when the recorded sentence contained a boundary in the preferred location, both when it was the only boundary and when two boundaries were presented.

\section{Discussion}

Participants in Experiment 1 consistently repeated sentences with the same intonational phrasing of the recorded sentences, producing more intonational boundaries in the dispreferred and preferred boundary locations when the recorded sentences included boundaries in these locations. This is the first evidence that speakers can retain and reproduce intonational phrasing information for these sentences, at least for the brief interval between presentation of the recorded sentences and their own reconstructions.

It is interesting to note that these repetition effects were observed at both boundary locations. In particular, participants did not seem disturbed by the presence of an intonational boundary in a dispreferred location and were willing to produce a boundary in this location after hearing one. We can therefore infer that processing of these sentences was not substantially disrupted by the presence of a dispreferred boundary. In fact, across all participants, more trials in the no-boundaries condition were removed due to
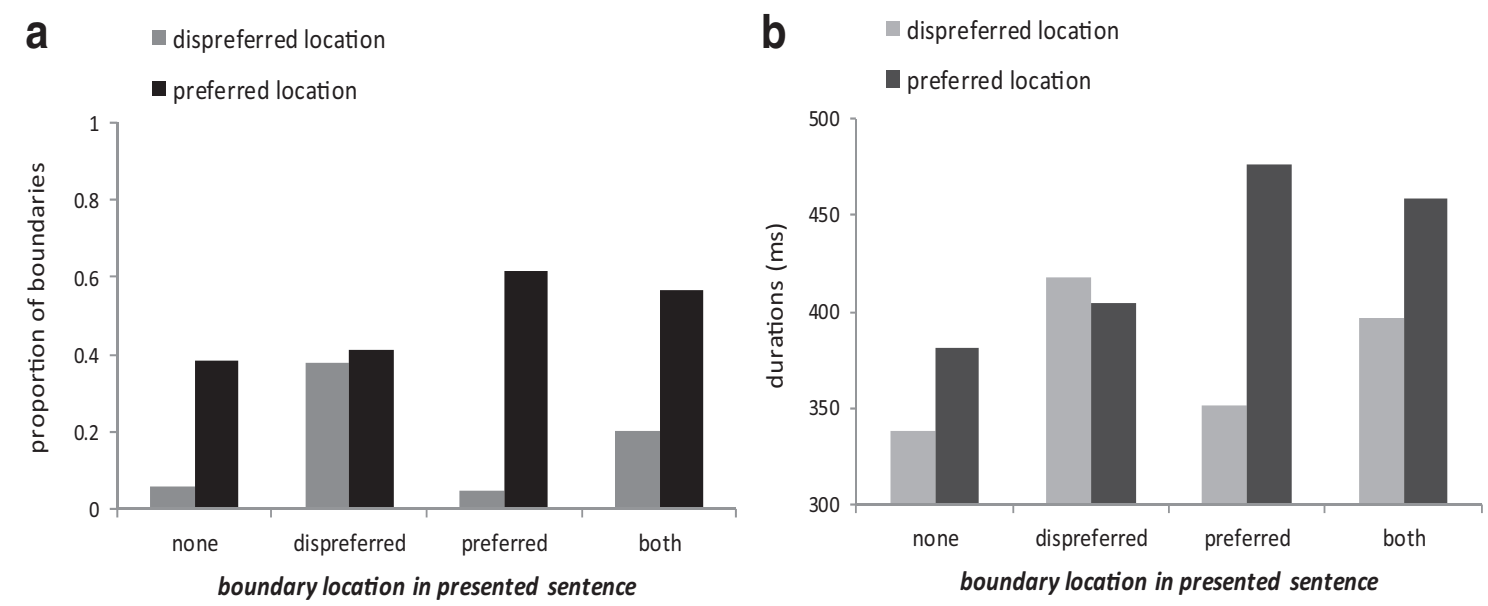

Figure 3. Proportion of perceived boundaries (Panel a) and word-and-pause durations (Panel b) at the dispreferred and preferred boundary locations in repeated sentences in Experiment 1. The $x$-axis shows the location of boundaries in the recorded prime sentences. 
incorrect reproduction than in the dispreferred boundary condition (78 and 73 sentences were removed in the no-boundaries condition and dispreferred boundary condition, respectively, for the boundary perception analysis, and 182 and 148 for the word-and-pause duration analysis); a dispreferred boundary was no more disruptive than the absence of a boundary.

However, the results of this experiment do not necessarily imply that speakers retained an abstract intonational phrase structure or that this intonational phrase structure could influence the intonational phrasing of a subsequent sentence, creating a priming effect analogous to a syntactic priming effect. For example, these results may also be explained by a mechanism in which the acousticphonetic properties of individual lexical items in the recorded sentence are briefly retained until the sentence is reproduced. Thus, rather than priming an abstract intonational phrase structure, participants may have simply remembered how to reproduce a particular sequence of lexical items.

Our analysis of participants' reproductions of sentences with wording changes makes this possibility less tenable. When repeating back the sentences, participants sometimes made slight changes in sentence wording, for example, by adding words (screened the samples to screened a few samples) or changing content words to words similar in meaning and sound (dolphin who tossed the ball to dolphin who touched the ball) or to words with different meanings and different sounds (billygoat that roamed the cliffs to billygoat that roamed the store). Comparing the distribution of perceived pauses across conditions in this subset of the data revealed the same pattern that was found in the larger data set: Participants were more likely to produce an intonational phrase boundary in a sentence location if the recording of the sentence they heard had a boundary in that location (this effect was significant at the dispreferred location and marginally significant at the preferred location). Thus, although speakers attempted to reconstruct sentences verbatim (as expected in a memory task), this result suggests that participants were not merely retaining verbatim lexical-acoustic information of the recorded sentences: Retention of intonational phrasing was robust enough to survive minor changes in surface form.

We next tested whether the intonational phrase structure of sentences presented on a prime trial would generalize to a novel sentence produced on the next trial (i.e., a target trial). Using generalization as a key test of abstractness and longevity of priming, Experiments 2 and 3 tested whether speakers would produce novel, unrelated but syntactically similar sentences with the intonational phrasing of recently experienced primes. In short, can the placement of intonational phrase boundaries heard in one sentence influence the placement of these boundaries in a new sentence?

\section{Experiment 2}

Experiment 2 used a prime-target paradigm to examine the effect of a recently heard and repeated sentence (the listen-andrepeat prime) on speakers' production of a second, unrelated sentence (the target). Participants heard and immediately repeated prime sentences, which had the same intonational phrasing manipulation as in Experiment 1. Prime trials were immediately followed by target trials, where participants silently read a sentence printed on a computer screen and then repeated it out loud from memory when the word REPEAT appeared on the screen. Since the target sentences appeared in written form, they were more neutral than recorded sentences with respect to intonational phrase structure.

If the repetition of intonational boundaries observed in Experiment 1 is due to priming of an abstract formulation stage, then the retained intonational phrasing from the listen-and-repeat prime sentences should influence the intonational phrasing of a novel target sentence that is presented without overt intonational phrasing. Thus, higher rates of production of boundaries in the read-andrepeat target sentences in the same locations as in the listen-and-repeat primes would indicate priming of intonational phrase structure. On the other hand, finding no influence of the prime sentences' intonational phrasing on boundary placement in the target sentences would support the notion that intonational phrase structure is not as readily primeable as syntactic structure, implying a lack of a separate processing stage where speakers plan an abstract intonational phrase structure.

\section{Method}

Participants. Sixty-four students from the University of Illinois, all native speakers of English, participated for course credit.

Materials. The materials were the same as in Experiment 1. Sentences were paired to create prime-target pairs where the two sentences had the same syntactic structure (both were either main clause sentences or relative clause sentences). The prime sentences were always listen-and-repeat trials, while the target sentences were always silent read-and-repeat trials. The order of these sentences was counterbalanced, such that each sentence in a primetarget pairing served as a prime and as a target on different lists (referred to as sentence order in Table 3). As in Experiment 1, prime sentences were presented in one of four conditions, creating eight lists of stimuli after counterbalancing the assignment of sentences to prime and target positions.

Three filler sentences, similar to those used in Experiment 1, intervened between any two prime-target pairs. Filler sentences were randomly assigned to be presented either as listen-and-repeat trials or read-and-repeat trials, and this assignment remained the same on all eight lists.

Procedure. Participants were told that on each trial they would either have to listen to a recorded sentence or silently read a printed sentence on the computer screen and then would be prompted to repeat these sentences back from memory into a microphone. On trials with auditorily presented sentences, participants saw the word LISTEN, which remained on the screen while they heard a sentence. At sentence offset, they saw the word REPEAT, which prompted them to repeat back the sentence aloud into a microphone. After repeating the sentence, they pressed the spacebar to advance to the next trial. On trials with printed sentences, participants first saw the word $R E A D$ on the computer screen for $1 \mathrm{~s}$, followed by a sentence that remained on the screen for an amount of time proportional to sentence length $(50 \mathrm{~ms}$ multiplied by the number of words in the sentence) to prevent different amounts of rehearsal time between longer and shorter sentences. Participants first read this sentence silently and then repeated it out loud from memory when the word REPEAT appeared on the screen. Following production, the participant again pressed the spacebar to move on to the next trial. 
Table 3

Results of Analyses of Perceived Boundary Production and Word-and-Pause Durations in the Dispreferred and Preferred Locations of Prime and Target Sentences Repeated in Experiment 2

\begin{tabular}{|c|c|c|c|c|c|c|}
\hline \multirow[b]{2}{*}{ Predictor } & \multicolumn{3}{|c|}{ Boundary production } & \multicolumn{3}{|c|}{ Word-and-pause durations } \\
\hline & Estimate & $S E$ & $z$ value & Estimate & $S E$ & $t$ value \\
\hline & \multicolumn{3}{|c|}{ Model a } & \multicolumn{3}{|c|}{ Model b } \\
\hline \multicolumn{7}{|l|}{ Dispreferred boundary location } \\
\hline Intercept & -2.67 & 0.27 & $-9.84^{*}$ & 345 & 15 & $23.39^{*}$ \\
\hline Sentence order (prime vs. target) & 0.10 & 0.30 & 0.34 & 19 & 7 & $2.72^{*}$ \\
\hline \multicolumn{7}{|l|}{ Condition contrasts } \\
\hline No pauses vs. dispreferred pause & 2.15 & 0.26 & $8.19^{*}$ & 65 & 8 & $8.08^{*}$ \\
\hline No pauses vs. preferred pause & -0.66 & 0.36 & -1.86 & 0 & 7 & -0.03 \\
\hline No pauses vs. both pauses & 1.30 & 0.27 & $4.87^{*}$ & 44 & 7 & $6.33^{*}$ \\
\hline \multicolumn{7}{|l|}{ Interactions with sentence position } \\
\hline No pauses vs. dispreferred pause & -1.76 & 0.38 & $-4.66^{*}$ & -65 & 10 & $-6.80^{*}$ \\
\hline No pauses vs. preferred pause & 0.83 & 0.45 & 1.83 & -7 & 10 & -0.73 \\
\hline \multirow[t]{2}{*}{ No pauses vs. both pauses } & -0.94 & 0.38 & $-2.46^{*}$ & -49 & 9 & $-5.20^{*}$ \\
\hline & \multicolumn{3}{|c|}{ Model c } & \multicolumn{3}{|c|}{ Model d } \\
\hline \multicolumn{7}{|l|}{ Preferred boundary location } \\
\hline Intercept & 0.21 & 0.22 & 0.97 & 398 & 17 & $22.74^{*}$ \\
\hline Sentence order (prime vs. target) & 0.32 & 0.19 & 1.72 & 14 & 9 & 1.60 \\
\hline \multicolumn{7}{|l|}{ Condition contrasts } \\
\hline No pauses vs. dispreferred pause & -0.12 & 0.19 & -0.64 & 18 & 9 & $2.00^{*}$ \\
\hline No pauses vs. preferred pause & 1.00 & 0.20 & $5.08^{*}$ & 50 & 9 & $5.50^{*}$ \\
\hline No pauses vs. both pauses & 0.90 & 0.20 & $4.63^{*}$ & 46 & 9 & $5.16^{*}$ \\
\hline \multicolumn{7}{|l|}{ Interactions with sentence position } \\
\hline No pauses vs. dispreferred pause & -0.11 & 0.26 & -0.40 & -21 & 12 & -1.65 \\
\hline No pauses vs. preferred pause & -1.07 & 0.27 & $-3.98^{*}$ & -36 & 12 & $-2.91^{*}$ \\
\hline \multirow[t]{2}{*}{ No pauses vs. both pauses } & -1.07 & 0.27 & $-4.03^{*}$ & -43 & 12 & $-3.47^{*}$ \\
\hline & \multicolumn{3}{|c|}{ Model e } & \multicolumn{3}{|c|}{ Model f } \\
\hline Targets only: Dispreferred boundary location & & & & & & \\
\hline Intercept & -2.55 & 0.25 & $-10.05^{*}$ & 363 & 14.37 & $25.23^{*}$ \\
\hline \multicolumn{7}{|l|}{ Condition contrasts } \\
\hline No pauses vs. dispreferred pause & 0.39 & 0.27 & 1.44 & 1.35 & 6.55 & 0.21 \\
\hline No pauses vs. preferred pause & 0.17 & 0.28 & 0.61 & -5.99 & 6.47 & -0.93 \\
\hline \multirow[t]{2}{*}{ No pauses vs. both pauses } & 0.35 & 0.27 & 1.28 & -4.19 & 6.47 & -0.65 \\
\hline & \multicolumn{3}{|c|}{ Model g } & \multicolumn{3}{|c|}{ Model h } \\
\hline \multicolumn{7}{|l|}{ Targets only: Preferred boundary location } \\
\hline Intercept & 0.51 & 0.19 & $2.63^{*}$ & 411 & 17.03 & $24.13^{*}$ \\
\hline \multicolumn{7}{|l|}{ Condition contrasts } \\
\hline No pauses vs. dispreferred pause & -0.24 & 0.18 & -1.33 & -3.53 & 8.42 & -0.42 \\
\hline No pauses vs. preferred pause & -0.06 & 0.18 & -0.36 & 16.06 & 9.59 & $1.68^{\dagger}$ \\
\hline No pauses vs. both pauses & -0.16 & 0.18 & -0.88 & 2.94 & 8.58 & 0.34 \\
\hline
\end{tabular}

Note. Models $\mathrm{b}$ and $\mathrm{f}$ include random by-participant slopes for the condition factor.

${ }^{\dagger} p<.10 .{ }^{*} p<.05$.

A practice block comprised of four listen-and-repeat and four read-and-repeat sentences in a pseudorandom order was added to the beginning of the experiment.

Scoring and analyses. The scoring was identical to Experiment 1 and left 2,320 trials (91\% of all trials) where speakers repeated the sentences either verbatim or with minor wording changes for the analysis of perceived pauses. For the analysis of word-and-pause durations, trials with minor word changes and with pauses longer than three standard deviations away from the grand mean were excluded, leaving 1,921 trials (or $75 \%$ of all trials) for analysis.

\section{Results}

Production of pauses in the dispreferred location. The analysis of perceived pauses at the dispreferred sentence region revealed a significant interaction between prime condition and sentence order (prime vs. target; Table 3, Model a). When the listen-and-repeat prime sentences contained boundaries at the dispreferred location, participants tended to produce boundaries at that sentence location. This was true whether there was only a boundary at the dispreferred location or boundaries at both sentence locations (see Figure 4a), replicating the findings from Experiment 1 .

The target sentences however, were not significantly affected by the priming manipulation at the dispreferred sentence location (see Table 3, Model e). Participants repeated back sentences that they read silently without producing a pattern of boundaries analogous to what they heard on the immediately preceding prime trial (see Figure $4 \mathrm{c}$ ). This suggests that priming of intonational phrase structure does not persist to a novel target 


\section{PRIMES}
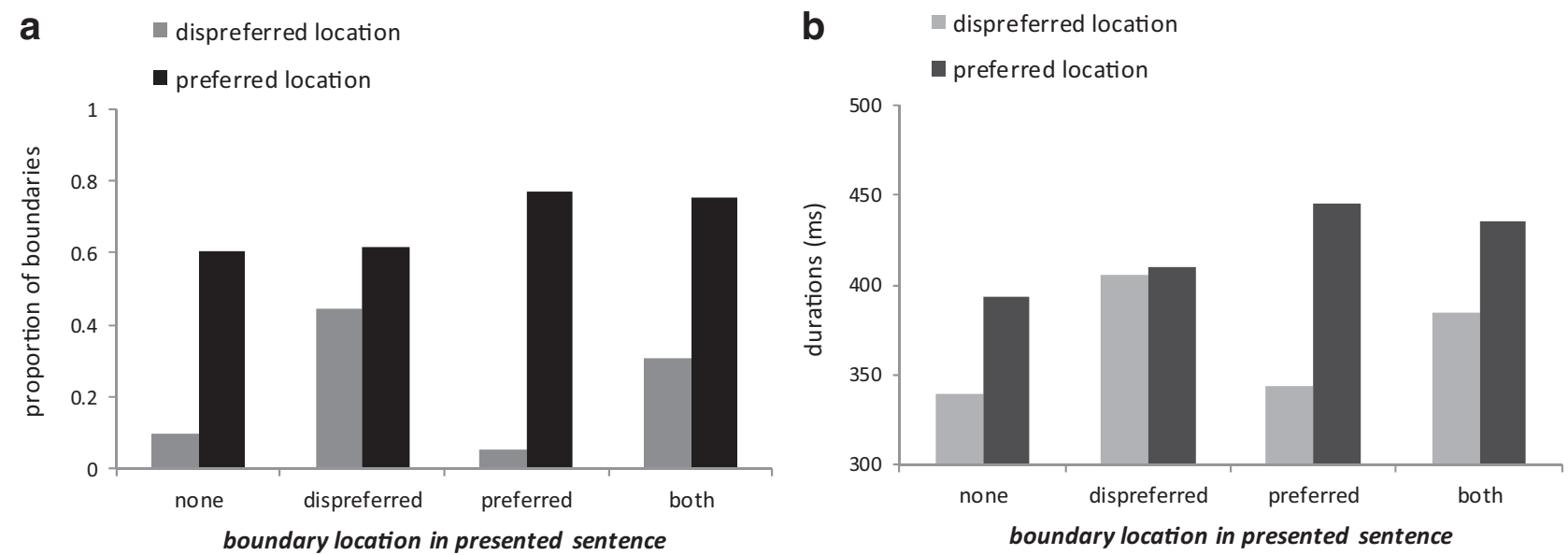

\section{TARGETS}
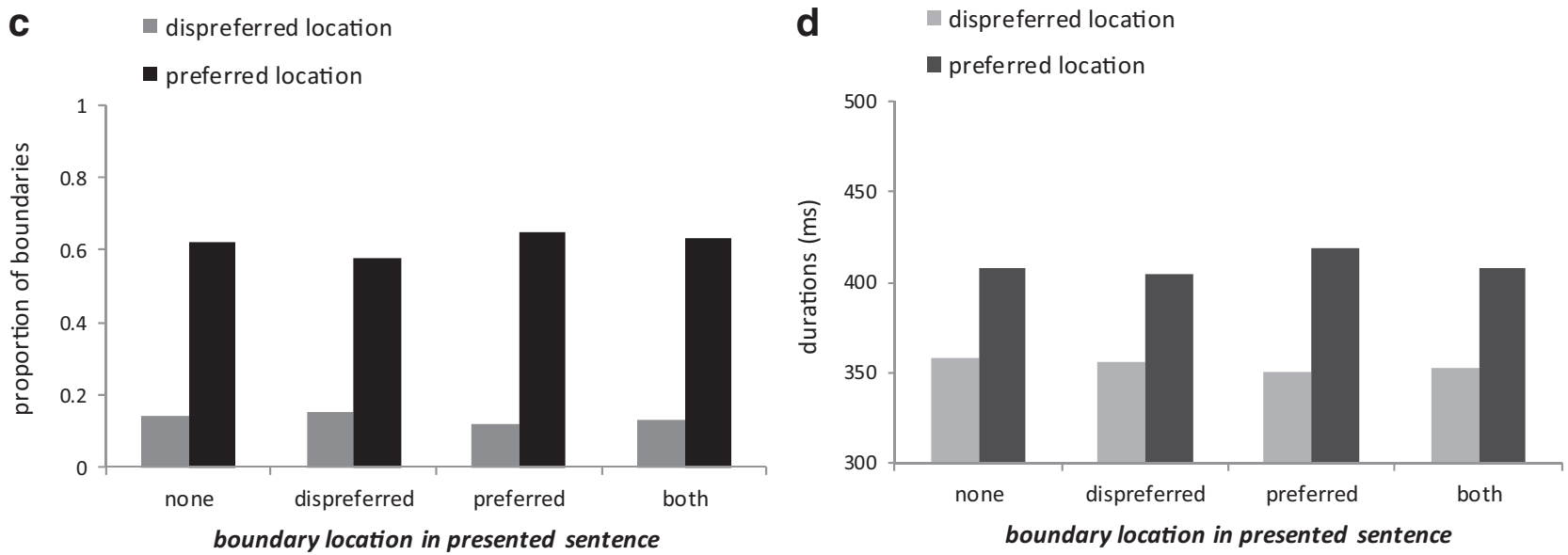

Figure 4. Proportion of perceived boundaries and word-and-pause durations at the dispreferred and preferred boundary locations in Experiment 2 in repeated prime sentences (Panels a and b) and repeated target sentences (Panels $\mathrm{c}$ and $\mathrm{d}$ ). The $x$-axis shows the location of boundaries in the recorded prime sentences.

sentence with the same syntactic structure but different words and meaning.

The analysis of word-and-pause durations showed the same pattern on prime trials, with speakers repeating both types of prime sentences with the intonational phrasing of the original recorded sentences: Word-and-pause durations in the dispreferred location were longer when the original sentences contained a boundary in this location or contained both boundaries than in the control condition (see Figure 4b). Production of the target sentences, however, was not affected by the intonational phrase structure of the primes, as word-and-pause durations did not differ across conditions (see Figure 4d and Table 3, Model f). This produced a reliable interaction between prime condition and sentence order (see Table 3, Model b).

Production of boundaries in the preferred location. Production of boundaries at the preferred sentence location (after the noun phrase) also resulted in an interaction of prime condition with sentence order (see Table 3, Model c). Participants tended to repeat prime sentences with a boundary at the preferred location when they were primed with a boundary at that location, but this pattern was not found for the target sentences (see Figures $4 \mathrm{a}, 4 \mathrm{c}$; Table 3, Model g). Thus, as with boundaries produced at the syntactically dispreferred location, there was no evidence to suggest that hearing a boundary at the preferred location in the prime sentence increased the likelihood that a participant would produce a boundary at that syntactic location in a subsequent novel sentence.

Similarly, word-and-pause durations in the preferred location in prime sentences were influenced by the intonational phrase structure of the primes, as in Experiment 1, but no differences were observed for target sentences across conditions (see Figures $4 \mathrm{~b}, 4 \mathrm{~d}$, and Table 3, Model $\mathrm{h}$ ). This resulted in a reliable interaction between prime condition and sentence order (see Table 3, Model d). 


\section{Discussion}

The results obtained with prime sentences replicated the pattern seen in Experiment 1. However, participants' production of target sentences did not differ across conditions: Participants produced boundaries in the dispreferred and preferred locations at similar rates and with similar durations regardless of the intonational phrase structure of the primes. This result provides converging evidence with Experiment 1 that participants are sensitive to and retain intonational phrase boundary information in the sentences that they hear, but critically, this information does not appear to influence intonational phrasing in subsequent sentences.

Because syntactic priming can be elicited reliably in similar paradigms (Konopka \& Bock, 2009; Potter \& Lombardi, 1998; Tooley \& Bock, 2013), the results suggest that abstract intonational phrase structure is likely not as primeable as syntactic structure. This discrepancy between the presence of syntactic priming under very similar conditions and the absence of intonational phrase structure priming in this study would be unexpected if an abstract intonational phrasing was generated at a separate stage during production. It is, however, consistent with the notion that intonational phrase structuring may not proceed at a separate level of representation; instead, intonational phrase structure may be constructed in concert with other levels of linguistic representation, such as syntax and semantics, and with the cognitive resources of the speaker.

An alternative explanation for the lack of priming on target trials is that the listen-and-repeat, read-and-repeat task used in this experiment created too large a temporal gap between repetition of the prime (listen-and-repeat) sentence and planning of the target (read-and-repeat) sentence. If the source of priming is activation in the prosodic processing system, then activation of an intonational phrase structure during production of the prime may have decayed before planning of the target utterance took place. Another possibility is that the intonational phrase structure that participants generated when repeating the prime sentence interfered with retention of the intonational phrasing to which they were exposed, thereby reducing or eliminating the likelihood of speakers reusing the primed intonational phrase structure in target sentences. To test the possibility that the lack of a reliable priming effect in target sentences was due to the time lag between prime and target trials or to interference inherent in the task, rather than the lack of persistence of an abstract prosodic structure, we also examined priming of intonational phrase structure in the same target sentences using a simplified task.

\section{Experiment 3}

Experiment 3 compared production of target sentences after passive exposure to primes. Speakers listened to prime sentences but did not have to repeat them out loud. The task on target trials was the same as in Experiment 2. If the priming of intonational phrase structure is relatively weak or short-lived, speakers may be more likely to repeat the intonational phrase structure of prime sentences on target trials when the temporal interval between presentation of a prime and production of the target sentence is reduced and no repetition of the prime sentence is required.

\section{Method}

Participants. Sixty-four adult native speakers of English participated for course credit or payment.

Materials. The materials and design were the same as in Experiment 2. Prime sentences were preceded by LISTEN prompts, but participants were not asked to repeat these sentences out loud from memory. Target sentences were again preceded by the word $R E A D$, and participants were asked to repeat these sentences upon seeing the word REPEAT on the screen.

To ensure that participants paid attention to the sentences preceded by a LISTEN prompt, the task on filler trials was manipulated to make sentence repetition on any given trial less predictable: Most of the READ fillers were not followed by a REPEAT prompt, and most of the LISTEN fillers were followed by a prompt. Thus, overall, participants received 24 trials where they listened to a sentence and repeated it out loud and 24 trials where they did not have to repeat the sentence. They also received 24 trials where they silently read a sentence and repeated it out loud and 25 trials where they did not have to repeat the sentence.

Procedure. On each trial, participants either listened to a recorded sentence or read a sentence printed on a computer screen. On some of these trials, they were then prompted to repeat out loud the sentence that they heard or saw. Participants were instructed to read and listen to all the sentences carefully, as they would not be told in advance which sentences they would be asked to repeat. At the end of each trial, participants pressed a key to continue to the next trial.

Scoring. The scoring was identical to Experiments 1 and 2 and left 1,116 trials ( $87 \%$ of all trials) for the analysis of perceived boundaries, including sentences reconstructed verbatim or reconstructed with minor wording changes. For the analysis of word-andpause durations, trials with minor word changes and with boundaries longer than three standard deviations away from the grand mean were excluded, leaving 890 trials (or $70 \%$ of all trials) for analysis.

\section{Results and Discussion}

The analysis of perceived boundaries revealed a pattern remarkably similar to that seen for the target sentences in Experiment 2. Prime condition was not a significant predictor of boundary location for either the dispreferred or preferred sentence locations (see Figure 5a; Table 4, Models a and c). Similarly, word-and-pause durations in the dispreferred and preferred boundary locations did not differ across conditions (see Figure 5b; Table 4, Models b and d). Indeed, all differences between conditions were numerically very small, showing that participants' production of boundaries in target sentences was not influenced by the prosodic phrasing of recently heard prime sentences with the same syntactic structure.

These findings replicate the results observed for target sentences in Experiment 2 and support the hypothesis that intonational phrase structure is not readily primeable. Even when the demands of the task and the time interval between processing the prime and producing the target were reduced, there was no evidence that hearing an intonational phrase boundary at a particular location in a prime sentence made participants more likely to produce a boundary at that sentence location in a subsequent target sentence.

It is still possible that potential priming effects were eliminated due to interference from the internal intonational phrasing generated by the participants as they silently read the target sentences. However, this possibility rests on the assumption that internal 

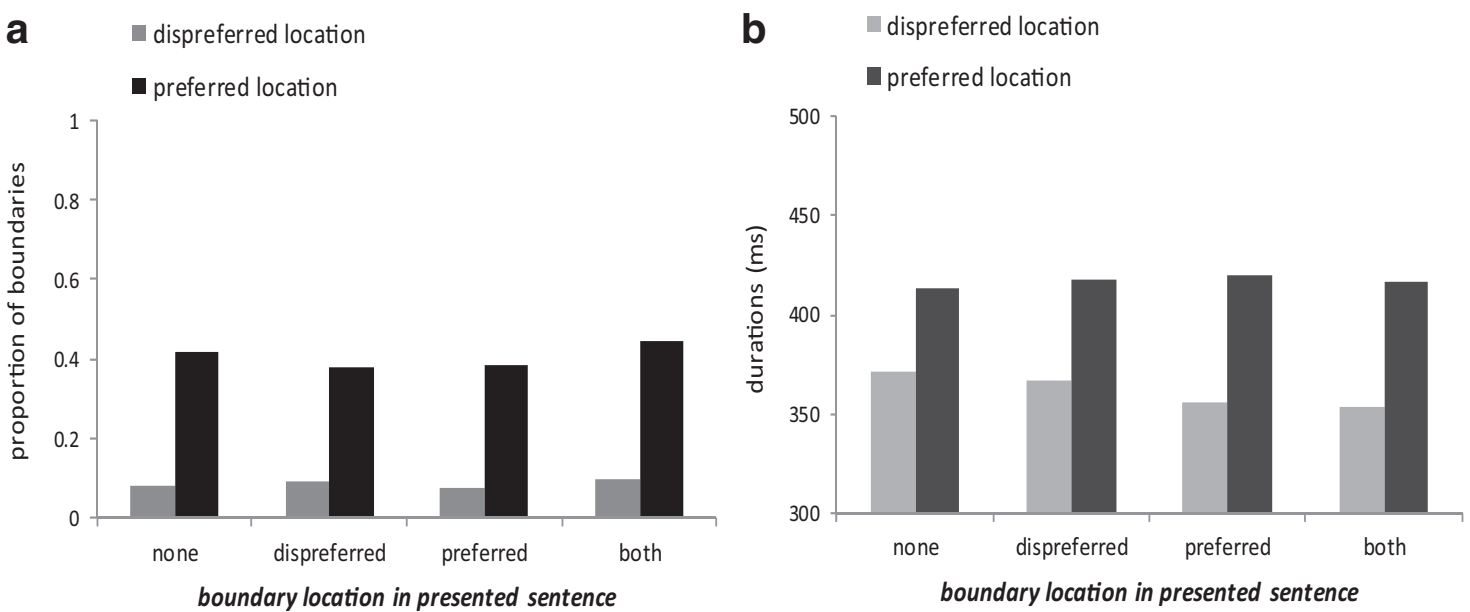

Figure 5. Proportion of perceived boundaries (Panel a) and word-and-pause durations (Panel b) at the dispreferred and preferred boundary locations in repeated target sentences in Experiment 3 . The $x$-axis shows the location of boundaries in the recorded prime sentences.

prosody would not be influenced by external prosodic information such as the intonational phrasing of the prime. Why would the internal prosody not likewise be primed? Prosodic priming should prime the internally generated prosody of the target sentence, which, in turn, should prime the prosody of the actual target production. Though this alternative explanation cannot be ruled out, it potentially raises more questions than it answers.

Comparing this finding to that of syntactic priming studies, the differences are stark. In nearly all priming studies in production, a single exposure to a syntactic structure is sufficient to influence the syntactic structure of a subsequent sentence (Bock, 1986; Pickering \& Branigan, 1998; and others), even when this sentence is produced after 10 intervening trials from the time of original exposure to the structure (Bock et al., 2007; Bock \& Griffin, 2000). Here, the same amount of exposure to a sentence with a specific intonational phrase structure produced no discernible effect on the intonational phrase structure of a subsequent sentence. If both syntactic and intonational phrase structures were assembled in similarly abstract processing stages, then we would expect both of these types of representations to be primeable under similar experimental conditions. The results from this experiment thus do not support such a unique processing stage for the planning of intonational phrase structure.

\section{General Discussion}

In three experiments, we investigated whether intonational phrase structure is as amenable to priming as syntactic structure has proven to be (Bock, 1986; Pickering \& Branigan, 1998; see Pickering \& Ferreira, 2008, for a recent review). Experiment 1

Table 4

Results of Analyses of Perceived Boundary Production and Word-and-Pause Durations in the Dispreferred and Preferred Locations of Target Sentences Repeated in Experiment 3

\begin{tabular}{|c|c|c|c|c|c|c|}
\hline \multirow[b]{2}{*}{ Predictor } & \multicolumn{3}{|c|}{ Boundary production } & \multicolumn{3}{|c|}{ Word-and-pause durations } \\
\hline & Estimate & $S E$ & $z$ value & Estimate & $S E$ & $t$ value \\
\hline & \multicolumn{3}{|c|}{ Model a } & \multicolumn{3}{|c|}{ Model b } \\
\hline \multicolumn{7}{|l|}{ Dispreferred boundary location } \\
\hline Intercept & -2.81 & 0.28 & $-10.21^{*}$ & 370 & 15 & $24.85^{*}$ \\
\hline \multicolumn{7}{|l|}{ Condition contrasts } \\
\hline No pauses vs. dispreferred pause & 0.19 & 0.32 & 0.60 & 2 & 8 & 0.28 \\
\hline No pauses vs. preferred pause & -0.11 & 0.34 & -0.31 & -9 & 8 & 1.07 \\
\hline \multirow[t]{2}{*}{ No pauses vs. both pauses } & 0.21 & 0.32 & 0.67 & -9 & 8 & 1.13 \\
\hline & \multicolumn{3}{|c|}{ Model c } & \multicolumn{3}{|c|}{ Model d } \\
\hline \multicolumn{7}{|l|}{ Preferred boundary location } \\
\hline Intercept & -0.44 & 0.20 & $-2.15^{*}$ & 420 & 18 & $22.76^{*}$ \\
\hline \multicolumn{7}{|l|}{ Condition contrasts } \\
\hline No pauses vs. dispreferred pause & -0.15 & 0.19 & -0.79 & 7 & 10 & 0.69 \\
\hline No pauses vs. preferred pause & -0.16 & 0.19 & -0.87 & 3 & 10 & 0.31 \\
\hline No pauses vs. both pauses & 0.17 & 0.19 & 0.36 & 6 & 10 & 0.56 \\
\hline
\end{tabular}

${ }^{*} p<.05$. 
revealed that participants reproduced the intonational phrasing of sentences that they had just heard. This was true for cases in which the intonational phrasing supported the syntactic phrasing (boundaries at preferred syntactic locations), those in which it did not (boundaries at dispreferred syntactic locations), and those in which there was more than one pause in a sentence (boundaries at both preferred and dispreferred syntactic locations). However, the intonational phrasing that participants heard and repeated did not influence where they produced intonational phrase boundaries in a subsequent novel sentence: There was no evidence of priming both when participants were asked to repeat back a prime sentence before reading and repeating a novel target sentence (Experiment 2) and when participants did not repeat the prime (Experiment 3). Importantly, the two types of analyses that we report here- that is, the analysis of perceived boundaries and the analysis of word-andpause durations-provided converging evidence in all experiments.

These findings suggest that intonational phrase structure is not as readily primeable as syntactic structure (Bock, 1986, and others) and are not consistent with an abstract intonational phrase structuring process. Although future work will be needed to specify the exact nature of intonational phrase production, these data point toward a production system in which intonational phrase boundary production likely interacts with other linguistic levels such as syntax, semantics, and discourse, as well as performance constraints such as planning processes, speech rate, and even other levels of prosodic representation. Boundaries may be inserted into the sentence as needed by these other levels of representation, but an abstract level of representation in which the entire intonational phrasing of a sentence is abstractly represented and planned may not exist.

Another possibility is that an abstract level of intonational phrase boundary production exists but is simply not amenable to priming. It could be that intonational boundaries are intimately linked to production processes, syntactic structure, and information structure and are therefore purposely insulated from activation from the environment, leaving boundaries to serve at the pleasure of other levels of a speaker's linguistic representation. This would mean that there are levels of linguistic representation that prime and others that do not. This implies that, contrary to alignment theory (Garrod \& Pickering, 2004), priming may not be as ubiquitous as previously thought. Such a distinction might provide plausible constraints on theories that have attempted to explain priming in language, including alignment theory. For example, syntactic representations and prosodic representations arguably differ in the degree to which they are reliable signals of meaning: Syntactic structures impart information that is critical for conveying who did what to whom, whereas the intonational boundary structure of the same sentences is less likely to do so. Thus, the functional value of alignment at these levels of representation may differ or may depend heavily on context. The role of possible differences in the communicative value of distinct levels of representation in priming remains to be explored.

\section{Differences in Priming Between Prosodic and Syntactic Structure}

In similar prime-target paradigms, the persistent repetition of syntactic structure across unrelated sentences has provided evi- dence that the syntactic processing of one sentence influences the syntactic processing of a subsequent sentence (Bock, 1986; Branigan et al., 2000; Garrod \& Pickering, 2004; Pickering \& Branigan, 1998). Priming of syntactic structure is evident within the productions of one person (Bock, 1986; Pickering \& Branigan, 1998) as well as across individuals in a conversational setting (Branigan et al., 2000). The target sentence is also affected to the same extent whether the priming exposure involves comprehension or production processing (Bock et al., 2007; Tooley \& Bock, 2013) and whether the primes and targets occur in succession or are separated by a number of intervening sentences (Bock et al., 2007; Bock \& Griffin, 2000; Hartsuiker, Bernolet, Schoonbaert, Speybroecke, \& Vanderelst, 2008).

In contrast, the current study generated no evidence to suggest that the intonational phrase structure of one sentence can influence the produced intonational phrase structure of a subsequent target sentence. While we do not draw a direct comparison between syntactic priming effects and intonational phrase structure priming effects in these studies, we note that the striking difference between reliable syntactic priming effects and nonreliable, numerically feeble intonational phrase structure priming effects suggests a qualitative difference in the way speakers formulate these two types of structures. In short, when priming of intonational phrase structure is assessed in the same kind of experimental setting that normally yields priming of syntactic structure, intonational phrase structure does not appear to transfer from sentence to sentence.

There are two reasons for thinking that differences between syntactic priming and intonational phrase priming are warranted. First, Experiments 1 and 2 provide firm evidence that our participants were sensitive to and could retain the intonational phrasing that they heard in the experimental sentences, so it is unlikely that the priming manipulation was too weak to create processing differences across conditions. Second, the results from the first two experiments (with listen-and-repeat prime sentences) also suggest that our dependent measure was sensitive enough to detect possible repetition effects. Furthermore, if we assume a small effect size for intonational phrase structure priming (as is the case for syntactic priming), say, 0.15 , then to achieve a desired power level (i.e., the probability of correctly rejecting the null hypothesis) of 0.85 , using an alpha level of 0.05 , we would need to test 62 participants (as calculated with GPower, assuming multiple logistic regression estimating three parameters ${ }^{2}$; Mayr, Erdfelder, Buchner, \& Faul, 2007). Both Experiments 2 and 3 meet the required level of participants and thus should have sufficient power to detect an effect, yet there was not even a trend in the data providing evidence for transfer of intonational phrasing from prime to target sentences.

Another way to assess our confidence in the null results is by calculating a Bayes factor. A Bayes factor is a way to estimate how well the data support a particular theoretical prediction, as well as the null hypothesis, based on a planned comparison. In this case,

\footnotetext{
${ }^{2}$ GPower does not, at present, provide power estimates for mixedeffects models, so we report the estimate for fixed-effects models, though we acknowledge that this estimate could vary for the analyses we used. To help address this, we conducted a combined targets analysis using the target sentences from Experiments 2 and 3. This analysis yielded no significant effect of priming condition, though the sample size was twice the size $(n=128)$ of either Experiment 2 or 3 on its own.
} 
if priming were to occur on target trials, then we would expect a reliable mean difference of word-and-pause durations between the baseline (no boundary) condition and the preferred boundary condition (at the preferred boundary location), as well as a difference between the baseline condition and the dispreferred boundary condition (at the dispreferred boundary location). In order to estimate the mean difference and standard error of these effects, we conducted by-participant and by-item $t$ tests on the duration measure for each of these comparisons in target sentences from Experiments 2 and 3 combined. To compare the magnitude of these effects against an empirically motivated range of values indicative of priming, we then set our lower bound for an expected priming effect at zero and the upper bound at one standard deviation above the observed average duration difference in prime sentences across conditions in Experiment 2. We assumed all magnitudes of priming to be equally likely and calculated the Bayes factor over this interval. Applying these criteria yielded Bayes factors of .06 and .00 by participants and by items, respectively, for the dispreferred boundary location, as well as .38 and .12 by participants and by items, respectively, for the preferred boundary location. Bayes factors that are greater than 3 strongly support the theoretical prediction, and Bayes factors of less than .33 strongly support the null hypothesis. The obtained Bayes factors for our effects are therefore quite small and (all but one) give us good reason to interpret our null result as a true lack of a priming effect.

\section{Priming and Prosody}

These results are intriguing because a lack of priming of linguistic representation is the exception rather than the rule in psycholinguistics. Priming in the language system is indeed ubiquitous for several types of linguistic representations. For example, early studies of lexical priming (priming for individual words) showed that participants are faster at identifying a string of letters as a real word if they have recently processed a prime word that is related to the target word (e.g., Meyer \& Schvaneveldt, 1971). Similarly, priming has been observed for more complex representations, such as the construction of syntactic structures that support production of multiword utterances and the construction of a situation model. For example, when giving directions (e.g., "on the left"), participants are more likely to use an egocentric frame of reference (by referring to what is on their left) if their conversational partner has also adopted an egocentric frame of reference (Schober, 1993). Conversation partners also tend to show convergence with respect to the way they produce phonemes (Pardo, 2006). Set against this backdrop of robust priming effects, the lack of intonational phrase structure priming potentially points to theoretically relevant distinctions between levels of representation that are primeable and aspects of linguistic structure that are not.

Finally, this work does not preclude the possibility that other aspects of prosodic structure such as intonation, speech rate, affect, and pitch accenting might be amenable to priming. In fact, Jungers and Hupp (2009), for example, found that the speech rate of prime sentences influenced not only the rate at which participants repeated these sentences back but also performance on a subsequent picture task. Similar effects have been found in preschoolers (Hupp \& Jungers, 2009) and in participants working together to complete a map task (Finlayson, Lickley, \& Corley, 2010). In addition, Hirschberg (2011) has found convergence of intonational features like pitch in conversations. Thus, it is clear that the priming of some prosodic features is possible in different contexts. The fact that other aspects of prosody can be primed suggests that an abstract representation of these aspects of prosodic structure exists at some point in planning the structure of a sentence. However, the evidence suggests that this abstract prosodic plan may not include the production of intonational phrase boundaries.

\section{Conclusion}

In this study, we tested whether intonational phrasing is as readily primeable as other types of linguistic representations (such as syntax). In three experiments, we found that although participants are sensitive to and can briefly retain the intonational phrase structure information that they hear, this information does not bias their placement of intonational phrase boundaries in subsequent, novel sentences. Thus, unlike syntactic structure, exposure to a particular intonational phrase structure does not lead to production of sentences with that structure with greater frequency. We contend that this difference in priming across studies suggests that intonational phrase structures are not formulated during a planning stage that is separate from other sources of linguistic information.

\section{References}

Baayen, R. H., Davidson, D. J., \& Bates, D. M. (2008). Mixed-effects modeling with crossed random effects for subjects and items. Journal of Memory and Language, 59, 390-412. doi:10.1016/j.jml.2007.12.005

Bates, E., \& MacWhinney, B. (1982). Functionalist approaches to grammar. In E. Wanner \& L. Gleitman (Ed.), Language acquisition: The state of the art (pp. 173-218). New York, NY: Cambridge University Press.

Bock, J. K. (1982). Toward a cognitive psychology of syntax: Information processing contributions to sentence formulation. Psychological Review, 89, 1-47. doi:10.1037/0033-295X.89.1.1

Bock, J. K. (1986). Syntactic persistence in language production. Cognitive Psychology, 18, 355-387. doi:10.1016/0010-0285(86)90004-6

Bock, K., Dell, G. S., Chang, F., \& Onishi, K. H. (2007). Persistent structural priming from language comprehension to language production. Cognition, 104, 437-458. doi:10.1016/j.cognition.2006.07.003

Bock, K., \& Griffin, Z. M. (2000). The persistence of structural priming: Transient activation or implicit learning? Journal of Experimental Psychology: General, 129, 177-192. doi:10.1037/0096-3445.129.2.177

Bock, K., \& Levelt, W. (1994). Language production: Grammatical encoding. In M. A. Gernsbacher (Ed.), Handbook of psycholinguistics (pp. 945-984). San Diego, CA: Academic Press.

Branigan, H. P., Pickering, M. J., \& Cleland, A. A. (2000). Syntactic co-ordination in dialogue. Cognition, 75, B13-B25. doi:10.1016/S00100277(99)00081-5

Breen, M., Watson, D. G., \& Gibson, E. (2011). Intonational phrasing is constrained by meaning, not balance. Language and Cognitive Processes, 26, 1532-1562. doi:10.1080/01690965.2010.508878

Chang, F., Dell, G. S., \& Bock, K. (2006). Becoming syntactic. Psychological Review, 113, 234-272. doi:10.1037/0033-295X.113.2.234

Cleland, A. A., \& Pickering, M. J. (2003). The use of lexical and syntactic information in language production: Evidence from the priming of noun-phrase structure. Journal of Memory and Language, 49, 214-230. doi:10.1016/S0749-596X(03)00060-3

Cooper, W. E., \& Paccia-Cooper, J. (1980). Syntax and speech. Cambridge, MA: Harvard University Press.

Ferreira, F. (1991). Effects of length and syntactic complexity on initiation times for prepared utterances. Journal of Memory and Language, 30, 210-233. doi:10.1016/0749-596X(91)90004-4 
Ferreira, F. (1993). The creation of prosody during sentence production. Psychological Review, 100, 233-253. doi:10.1037/0033-295X.100.2 .233

Finlayson, I. R., Lickley, R. J., \& Corley, M. (2010, September). The influence of articulation rate, and the disfluency of others, on one's own speech. Paper presented at the DiSS-LPSS Joint Workshop, Tokyo, Japan.

Garrett, M. (1980). Levels of processing in sentence production. In B. Butterworth (Ed.), Language production (Vol. 1, pp. 177-220). New York, NY: Academic Press.

Garrod, S., \& Pickering, M. J. (2004). Why is conversation so easy? Trends in Cognitive Sciences, 8, 8-11. doi:10.1016/j.tics.2003.10.016

Gee, J., \& Grosjean, F. (1983). Performance structures: A psycholinguistic appraisal. Cognitive Psychology, 15, 411-458. doi:10.1016/00100285(83)90014-2

Griffin, Z. M., \& Bock, K. (2000). What the eyes say about speaking. Psychological Science, 11, 274-279. doi:10.1111/1467-9280.00255

Hartsuiker, R. J., Bernolet, S., Schoonbaert, S., Speybroecke, S., \& Vanderelst, D. (2008). Syntactic priming persists while the lexical boost decays: Evidence from written and spoken dialogue. Journal of Memory and Language, 58, 214-238. doi:10.1016/j.jml.2007.07.003

Hirschberg, J. (2011, September). Prosodic dimensions of entrainment in dialogue. Talk presented at Experimental and Theoretical Advances in Prosody 2, Montreal, Quebec, Canada.

Hupp, J. M., \& Jungers, M. K. (2009). Speech priming: An examination of rate and syntactic persistence in preschoolers. British Journal of Developmental Psychology, 27, 495-504. doi:10.1348/026151008X345988

Jaeger, T. F. (2008). Categorical data analysis: Away from ANOVAs (transformation or not) and towards mixed logit models. Journal of Memory and Language, 59, 434-446. doi:10.1016/j.jml.2007.11.007

Jungers, M. K., \& Hupp, J. M. (2009). Speech priming: Evidence for rate persistence in unscripted speech. Language and Cognitive Processes, 24, 611-624. doi:10.1080/01690960802602241

Konopka, A. E., \& Bock, K. (2009). Lexical or syntactic control of sentence formulation? Structural generalizations from idiom production. Cognitive Psychology, 58, 68-101. doi:10.1016/j.cogpsych.2008.05.002

Mayr, S., Erdfelder, E., Buchner, A., \& Faul, F. (2007). A short tutorial of GPower. Tutorials in Quantitative Methods for Psychology, 3, 51-59.

Meyer, D. E., \& Schvaneveldt, R. W. (1971). Facilitation in recognizing words: Evidence of a dependence upon retrieval operations. Journal of Experimental Psychology, 90, 227-234. doi:10.1037/h0031564

Nespor, M., \& Vogel, I. (1986). Prosodic phonology. Dordrecht, the Netherlands: Foris Publications.

Pardo, J. S. (2006). On phonetic convergence during conversational interaction. Journal of the Acoustical Society of America, 119, 2382-2393. doi:10.1121/1.2178720

Pickering, M. J., \& Branigan, H. P. (1998). The representation of verbs: Evidence from syntactic priming in language production. Journal of Memory and Language, 39, 633-651. doi:10.1006/jmla.1998.2592
Pickering, M. J., \& Ferreira, V. S. (2008). Structural priming: A critical review. Psychological Bulletin, 134, 427-459. doi:10.1037/0033-2909.134.3.427

Potter, M. C., \& Lombardi, L. (1998). Syntactic priming in immediate recall of sentences. Journal of Memory and Language, 38, 265-282. doi:10.1006/jmla.1997.2546

R Development Core Team. (2012). R: A language and environment for statistical computing. Vienna, Austria: R Foundation for Statistical Computing.

Schafer, A. (1997). Prosodic parsing: The role of prosody in sentence comprehension (Doctoral dissertation). University of Massachusetts at Amherst.

Schober, M. F. (1993). Spatial perspective-taking in conversation. Cognition, 47, 1-24. doi:10.1016/0010-0277(93)90060-9

Selkirk, E. O. (1984). Phonology and syntax: The relation between sound and structure. Cambridge, MA: MIT Press.

Shattuck-Hufnagel, S., \& Turk, A. E. (1996). A prosody tutorial for investigators of auditory sentence processing. Journal of Psycholinguistic Research, 47, 193-247. doi:10.1007/BF01708572

Silverman, K., Beckman, M., Pitrelli, J., Ostendorf, M., Wightman, C., Price, P., . . . Hirschberg, J. (1992, October). TOBI: A standard for labeling English prosody. Paper presented at the International Conference on Spoken Language Processing, Banff, Alberta, Canada.

Steedman, M. (1991). Structure and intonation. Language, 67, 260-296. Retrieved from http://www.jstor.org/stable/415107

Tooley, K. M., \& Bock, J. K. (2013). On the parity of structural persistence in language production and comprehension. Manuscript submitted for publication.

Tooley, K. M., Traxler, M. J., \& Swaab, T. Y. (2009). Electrophysiological and behavioral evidence of syntactic priming in sentence comprehension. Journal of Experimental Psychology: Learning, Memory, and Cognition, 35, 19-45. doi:10.1037/a0013984

Truckenbrodt, H. (1999). On the relation between syntactic phrases and phonological phrases. Linguistic Inquiry, 30, 219-255. doi:10.1162/ 002438999554048

Wagner, M., \& Watson, D. G. (2010). Experimental and theoretical advances in prosody: A review. Language and Cognitive Processes, 25, 905-945. doi:10.1080/01690961003589492

Watson, D., Breen, M., \& Gibson, E. A. (2006). The role of syntactic obligatoriness in the production of intonational boundaries. Journal of Experimental Psychology: Learning, Memory, and Cognition, 32, 10451056. doi:10.1037/0278-7393.32.5.1045

Watson, D., \& Gibson, E. (2004). The relationship between intonational phrasing and syntactic structure in language production. Language and Cognitive Processes, 19, 713-755. doi:10.1080/01690960444000070

Watson, D., \& Gibson, E. (2005). Intonational phrasing and constituency in language production and comprehension. Studia Linguistica, 59, 279300. doi:10.1111/j.1467-9582.2005.00130.x

Wheeldon, L., \& Lahiri, A. (1997). Prosodic units in speech production. Journal of Memory and Language, 37, 356-381. doi:10.1006/jmla.1997 .2517 


\section{Appendix}

\section{Sentences With Relative Clauses and Main Clauses Presented in Experiments 1-3}

Sentences with relative clauses

The apprentice who melted // the gold // had not slept in days.

The lender who approved // the loan // negotiated a good interest rate.

The clown who entertained // the children // wore a silly hat.

The witch who lived // in the old house // had three black cats.

The billygoat that roamed // the cliffs // was incredibly nimble.

The men who survived // the battle // huddled around the fire.

The dolphin that tossed // the ball // wanted a reward for his trick.

The jeweler who set // the stone // charged a large fee.

The guard who worked // the night shift // had a hard time staying awake.

The dancer who owned // the studio // was in excellent physical condition.

The professor who gave // the lecture // had a pronounced lisp.

The nurse who minded // the patients // had a kind smile.

The monkey that stole // the hat // refused to give it back.

The woman who watered // the flowers // enjoyed bright colors.

The queen who summoned // the painter // wanted a new mural.

The traveler who visited // the temple // loved exotic places.

The dog that pawed // the door // needed to be let out.

The violinist who performed // the solo // got a standing ovation.

The firefighter who stopped // the blaze // was given a medal.

The realtor who sold // the property // got a large commission.

Sentences with main clauses

The mobster shot // the men // for their disloyalty.

The duck splashed // the water // as it landed on the lake.

The botanist studied // the plant cells // with a powerful microscope.

The girl bought // new clothes // at the mall today.

The pianist rehearsed // the piece // for hours and hours.

The biologists freed // the whale // once it had fully healed.

The caterer set // the trays // on the long banquet table.

The gardener gave // the squash // to the family next door.

The movie star accepted // the role of Hamlet // from the director.

The engineer designed // the bridge // that crossed the bay.

The accountant reviewed // the material // before the certification exam.

The pigeon followed // the baby // around the park.

The valet requested // the shift // with the most business.

The brewer studied // the recipe // for the new pale ale.

The jogger patted // the dog // when he stopped for a drink.

The motorist bumped // the new car // while parking.

The minister asked // the congregation // to put money in the offering plate.

The cook divided // the soup // into four equal portions.

The scientist screened // the samples // for the deadly disease.

The miners struck // gold // after digging for a month.

Note. The locations of the dispreferred and preferred boundaries (first and second boundaries, respectively) are indicated with forward slashes. Auditory versions of these stimuli are available at http://labs.psychology.illinois .edu/CaLL/publications.html.

Received October 15, 2012

Revision received August 13, 2013

Accepted September 4, 2013 